\title{
Dynamic Cyclovergence during Vertical Translation in Humans
}

\author{
Itsaso Olasagasti, ${ }^{1}$ Christopher J. Bockisch, ${ }^{1,2,3}$ David S. Zee, ${ }^{4}$ and Dominik Straumann ${ }^{1}$ \\ Departments of ${ }^{1}$ Neurology, ${ }^{2}$ Otorhinolaryngology, and ${ }^{3}$ Ophthalmology, University Hospital Zurich, CH-8091 Zurich, Switzerland, and ${ }^{4}$ Department of \\ Neurology, Johns Hopkins Hospital, Baltimore, Maryland 21287
}

When humans are accelerated along the body vertical, the right and left eyes show oppositely directed torsional modulation (cyclovergence). The origin of this paradoxical response is unknown. We studied cyclovergence during linear sinusoidal vertical motion in healthy humans. A small head-fixed visual target minimized horizontal and vertical motion of the eyes and therefore isolated the torsional component. For stimuli between 1 and $2 \mathrm{~Hz}$ (near the natural range of head motion), the phase of cyclovergence with respect to inertial acceleration was $8.7 \pm 2.4^{\circ}$ (mean $\pm 95 \% \mathrm{CI}$ ) and the sensitivity (in degrees per second per $g$ ) showed a small but statistically significant increase with frequency. These characteristics contrast with those of cycloversion (conjugate torsion) during horizontal (interaural) inertial stimuli at similar frequencies. From these and previous results, we propose that cyclovergence during vertical translation has two sources, one, like cycloversion, from the low-frequency component of linear acceleration, and another, which we term dynamic cyclovergence, with high-pass characteristics. Furthermore, we suggest that this cyclovergence response in humans is a vestige of the response of lateral-eyed animals to vertical linear acceleration of the head.

\section{Introduction}

The orientation of the eye in the orbit has three degrees of freedom: two determine the direction of gaze and the third, torsion, describes the rotation about the line of sight. For behaviors that require stabilization of images on the fovea, a unique torsional position is observed for each gaze direction (Donder's law), and according to Listing's law, the rotation vectors describing eye positions in a head-centered frame lie in the displacement plane (DP) (Helmholtz, 1867; Haustein, 1989). This relationship is violated when dynamic visual stimuli generate a retinal flow with a torsional component (Cheung and Howard, 1991) or when the head rotates about a naso-occipital axis (e.g., Baarsma and Collewijn, 1975; Collewijn et al., 1985).

Although, in foveate species, compensation for translation does not require changes in torsion, cycloversion (Lichtenberg et al., 1982) and cyclovergence (de Graaf et al., 1996; Merfeld et al., 1996) are observed during linear displacements along the interaural (IA) and the vertical axes, respectively. Cycloversion arises because, without semicircular canal activation, the low-frequency components of the otolith signals are interpreted as reorientations with respect to gravity (Baarsma and Collewijn, 1975; Paige and Tomko, 1991; Angelaki, 1998; Raphan and Cohen, 2002;

Received Jan. 5, 2011; revised May 18, 2011; accepted May 20, 2011.

Author contributions: I.0., C.J.B., and D.S. designed research; I.0. performed research; I.O., C.J.B., D.S.Z., and D.S. analyzed data; I.O., C.J.B., D.S.Z., and D.S. wrote the paper.

This work was supported by the Swiss National Science Foundation; the Koetser Foundation for Brain Research; the Center for Integrative Human Physiology, University of Zurich; and the Leon Levy Foundation. We thank Albert Züger for technical assistance.

Correspondence should be addressed to Itsaso Olasagasti, Vestibulo-Oculomotor Laboratory, Department of Neurology, University Hospital Zurich, Frauenklinikstrasse 26, UHAL 26, CH-8091 Zurich, Switzerland. E-mail: miren.olasagasti@usz.ch.

DOI:10.1523/JNEUROSCI.0064-11.2011

Copyright $\odot 2011$ the authors $\quad 0270-6474 / 11 / 319991-07 \$ 15.00 / 0$
Merfeld et al., 2005; Zupan and Merfeld, 2005). So far, there is no explanation for cyclovergence during vertical translation.

Disconjugate torsional components also appear with changes in gravity load (de Graaf et al., 1996; Diamond and Markham, 1998; Clarke and Haslwanter, 2007) and lateral head roll tilts, in which both eyes counter-rotate in the same direction but with different amplitudes (Markham and Diamond, 2002; Pansell et al., 2003; Palla et al., 2006).

Here we wish to discern the origin of cyclovergence during vertical linear motion. We consider several hypotheses. Hypotheses 1 and 2 relate to known kinematical relations, while hypotheses 3 and 4 involve additional oculomotor signals. (1) Cyclovergence occurs because the DP of both eyes might show a small temporal divergence, which induces oppositely directed torsion with vertical eye movements. (2) DP might rotate temporally back and forth in response to vertical inertial acceleration. (3) Since the utricular maculae are not horizontal planes in the head and so are stimulated by vertical acceleration (Curthoys et al., 2009), the mechanism causing cycloversion during IA inertial stimulation could evoke cyclovergence during vertical inertial acceleration. (4) Cyclovergence is phylogenetically old. Rats, a lateral-eyed species, show a weak vertical eye response to inertial vertical acceleration (Hess and Dieringer, 1991). In frontal-eyed species, the same head-centered axis of rotation leads to cyclovergence.

To unravel these complexities of torsional motion of the eyes, we expanded on previous studies of cyclovergence in response to whole-body longitudinal sinusoidal motion. We used higherfrequency stimuli, closer to those experienced during walking (Moore et al., 2001); we measured the phase of cyclovergence, which was not reported before; and, in two of our three paradigms, inertial acceleration was earth-vertical, not earth- 
horizontal as in previous studies, and therefore the gravitoinertial vector (GIV) changed purely in magnitude.

Preliminary data has appeared in abstract form (Olasagasti et al., 2008).

\section{Materials and Methods}

\section{Equipment}

A six-degree-of-freedom motion platform (E-Cue $624 \times 1800$, FCS Control Systems) was used for all experiments. A chair was mounted on the platform, and the subject was secured with a four-point safety harness, with straps over the shoulders and thighs, and subjects held onto a horizontal bar to provide additional stability. A thermoplastic mask, molded for each subject, secured the head to the back of the chair. For experiments with the subject lying on their side, the same chair was rotated on its side and additional padding was inserted under the head and side of the body to increase stability.

Three-dimensional movements of both eyes were recorded with dualsearch coils manufactured by Skalar. Coils were calibrated in vitro on a gimbal system before each experiment (Straumann et al., 1995). A chairfixed coil frame that produced three orthogonal magnetic fields with frequencies of 80,96 , and $120 \mathrm{kHz}$ surrounded the subject's head. A digital signal processor (Primelec) computed a fast Fourier transform on the digitized search coil signal in real time to determine the voltage induced in the coil by each magnetic field. Eye position and motion platform signals were sampled at $1000 \mathrm{~Hz}$ and were digitized with 12 bit precision.

Fixation targets were $0.25^{\circ}$ diameter red light-emitting diodes, which could either be shown continuously or blinked ( $2 \mathrm{~s}$ off, $20 \mathrm{~ms}$ on).

\section{Sign convention and nomenclature}

We use a right-handed reference system to describe rotations and translations with positive directions defined as follows: naso-occipital toward the front ( $x$-axis), interaural toward the left ear ( $y$-axis), and vertical upward ( $z$-axis). For rotations, this corresponds to clockwise (as seen from the subject), downward, and leftward eye movements, respectively. As a result, the upward translation of the chair is positive but the upward eye rotation is negative.

Rotation vectors are defined in the standard manner, $\vec{r}=\tan (\theta / 2) \hat{u}$, where $\hat{u}$ is a unit vector representing the axis of rotation and $\theta$ is the angle of rotation. In the figures, to help visualization, we use a nonstandard representation in which $\vec{r}^{\prime}=\theta \hat{u}$.

The plane defined by Listing's law is defined in head coordinates, and therefore we report torsion in head coordinates. For far targets, the deviation of the optic axis, which defines the retinal torsion, and the nasooccipital axis, which defines head-based torsion, is small (about $2^{\circ}$ for a target straight ahead at a distance of $1.4 \mathrm{~m}$ ).

Since the otoliths, which function as inertial linear accelerometers, are equally stimulated by gravitational and linear acceleration, it is convenient to use the term gravitoinertial vector (GIV) to refer to their combined effect. If $\vec{g}$ is the gravitational vector and $\vec{a}$ represents the acceleration of the head in space, the force sensed by the otoliths in the noninertial frame of the head is proportional to $\vec{a}_{\mathrm{GIV}}=\vec{g}-\vec{a}$. When linear acceleration is earth-vertical, $\vec{g}$ and $\vec{a}$ are parallel and the GIV changes only in magnitude. This can be interpreted as a change in gravity load. For sinusoidal motion at a frequency $f$, the vertical component of linear acceleration is related to vertical displacement $\left[z_{H}(t)\right]$ by $a_{z}(t)=$ $-(2 \pi f)^{2} z_{H}(t)$; therefore, the effective gravity load is g-load $=g-$ $(2 \pi f)^{2} z_{H}(t)$. That is, effective gravity decreases as chair goes up and increases as the chair goes down. The magnitude of linear acceleration is expressed as a fraction of " $g$," the acceleration of an object in free fall ( 9.81 $\mathrm{m} / \mathrm{s}^{2}$ ), which sets a natural scale.

\section{Experimental paradigm}

Inertial stimuli were aligned with the participant's longitudinal axis in two whole-body orientations: sitting upright (UP) or lying $90^{\circ}$ right ear down (RED). In all cases, stimuli consisted of sinusoidal translations: earth-vertical in upright position and earth-horizontal in RED position.
Table 1. Summary of stimulus parameters

\begin{tabular}{|c|c|c|c|c|c|}
\hline \multicolumn{2}{|c|}{ Paradigm 1} & \multicolumn{2}{|c|}{ Paradigm 2} & \multicolumn{2}{|c|}{ Paradigm 3} \\
\hline$f(\mathrm{~Hz})$ & $a_{\text {peak }}(g)$ & $f(\mathrm{~Hz})$ & $a_{\text {peak }}(g)$ & $f(\mathrm{~Hz})$ & $a_{\text {peak }}(g)$ \\
\hline 1.5 & $0.42,0.50,0.57$ & 1 & $0.24,0.28,0.33$ & 1.5 & $0.45,0.53,0.62$ \\
\hline 1.75 & $0.44,0.55,0.66$ & 1.5 & $0.44,0.53,0.61$ & 1.75 & $0.47,0.59,0.71$ \\
\hline 2 & $0.43,0.56,0.70$ & 1.75 & $0.47,0.59,0.70$ & 2 & $0.45,0.60,0.75$ \\
\hline
\end{tabular}

During paradigms 1 and 2 , subjects were sitting upright and the net GIV changed only in magnitude. For paradigm 3 , subjects lay on their right side so that the head interaural axis was earth-vertical.

In a few of the recordings, we used a third 3D coil attached to a bite piece to measure residual head motion. Extraneous head rotation during vertical stimulation in upright position was $<1^{\circ}$ of amplitude.

Experiments were performed in a dark room, and the only source of light was a chair-fixed LED. In most of the experiments, the LED was constantly on to suppress horizontal and vertical eye movements, but we also presented a flashing LED to some subjects.

There were three stimulus paradigms, each with nine conditions (Table 1), three frequency values with three peak accelerations. The order of stimuli was randomized but was the same for every subject.

None of the volunteers that participated in this study had any known vestibular deficits.

Stimuli in upright position. Nine volunteers took part in these paradigms (five female, four male). Stimuli consisted of earth-vertical sinusoidal translations aligned with the participant's longitudinal axis. Consequently, the net gravitoinertial vector changed in amplitude but not in direction. Stimulus paradigm 1 (P1) included frequencies of 1.5, 1.75 , and $2 \mathrm{~Hz}$. In a follow-up paradigm, we ran stimulus paradigm 2 (P2), in which we shifted the frequency range to include $1 \mathrm{~Hz}$. For each frequency and peak acceleration the stimulus included 13 cycles. The LED was straight ahead at a distance of $1.43 \mathrm{~m}$ or $16 \mathrm{~cm}$ in front of the left eye in trials that control for target distance.

Stimuli in right ear down side position. Five participants ( 2 male, 3 female) lay on their side and underwent earth-horizontal inertial acceleration along their body vertical axis. Stimulus parameters were as indicated in the table under paradigm 3. The LED was constantly on in all cases. Because of the physical configuration of the apparatus, the LED was $80 \mathrm{~cm}$ straight ahead in RED position.

\section{Control paradigm}

The majority of our experiments had a fixation LED to cancel horizontal and vertical eye movements during translational motion. To test a possible contribution to cyclovergence from the pursuit system, which is involved in vestibulo-ocular reflex (VOR) cancellation, we tested three subjects with stimuli ranging from visual only to vestibular only. With a fixation distance of $120 \mathrm{~cm}$, we presented the following conditions: (1) visually enhanced linear VOR (space-fixed target on), (2) pure pursuit paradigm (chair stationary, laser dot moving with the amplitude and frequency of chair motion in vestibular stimulation), (3) linear VOR in complete darkness (fixation spot disappears during chair motion), and (4) VOR cancellation (chair-fixed fixation dot on). Additionally, we repeated the VOR cancellation condition with a fixation distance of $60 \mathrm{~cm}$. In Figure 4 and in the Results, these five paradigms are named C1 to C5.

In each of the five conditions, we presented two sinusoidal stimuli: one with an amplitude of $6.9 \mathrm{~cm}$ at $1.25 \mathrm{~Hz}$ and the other with an amplitude of $3.5 \mathrm{~cm}$ at $1.75 \mathrm{~Hz}$. In the conditions with chair translation, peak linear acceleration was $0.4 \times g$ for both.

\section{Data collection and analysis}

Three-dimensional eye movements were recorded binocularly using the dual scleral search coil technique. The resolution of the system is $0.1^{\circ}$. All the data were analyzed offline with custom scripts written in MATLAB. Voltage signals from the coils were transformed into rotation vectors and angular velocity was calculated. Time derivatives were calculated with a SavitzkyGolay filter of third order and 71 point size $(71 \mathrm{~ms})$ (Savitzky and Golay, 1964). Rotation vectors are defined in a chair-fixed frame; therefore, calculated angular velocity corresponds to the motion of the eye in the chair. Since the LED moved with the chair, vertical and horizontal eye movements in the chair frame directly reflect the success of gaze stabilization. 
We studied the conjugate (CON) and disconjugate (DIS) components of angular velocity, $\vec{\omega}_{\mathrm{CON}} \equiv\left(\vec{\omega}_{\mathrm{R}}+\vec{\omega}_{\mathrm{L}}\right) / 2$ and $\vec{\omega}_{\mathrm{DIS}} \equiv\left(\vec{\omega}_{\mathrm{R}}-\vec{\omega}_{\mathrm{L}}\right)$ (subscripts R and L refer to right and left eye, respectively). For the disconjugate component of torsional eye movements, we will use the terms cyclovergence and disjunctive or disconjugate torsion interchangeably.

For each experimental condition, 11 cycles were aligned (the first and last were discarded because they corresponded to fade-in and fade-out cycles of the motion platform) and the median angular velocity was calculated. The median was chosen because it is robust to outliers such as artifacts due to blinks and saccades. For each eye and each component of angular eye velocity, we obtained the median over a period and fit it to a sinusoid of the same frequency as the stimulus plus a constant.

$$
\omega(t)=A \cos (2 \pi f t)+B \sin (2 \pi f t)+C .
$$

The sinusoidal part can be represented by a vector with components $(A$, $B$ ) or through the amplitude $R$ and phase $\Phi, A \cos (2 \pi f t)+B \sin (2 \pi f t) \equiv$ $R \sin (2 \pi f t-\Phi)$, which are determined by the relations $R=\sqrt{A^{2}+B^{2}}$, $\sin (\Phi)=-A / \sqrt{A^{2}+B^{2}}$, and $\cos (\Phi)=-B / \sqrt{A^{2}+B^{2}}$. The parameter $C$ is an offset for any constant drift in angular velocity. Since $C$ was well below $1 \%$, we could ignore it.

During this cycle, chair linear velocity was $V \sin (2 \pi f t)$, and linear acceleration was $a \cos (2 \pi f t)=a \sin (2 \pi f t+\pi / 2)$. Therefore, $\Phi$ represents the phase lag of eye velocity relative to chair velocity and $\Phi+\pi / 2$ the phase lag with respect to acceleration.

Since stimuli include different peak accelerations, we will characterize responses with a normalized sensitivity measure $S=R / a$ with units of degrees per second per $g$ and the phase with respect to acceleration $\Phi^{\prime} \equiv$ $\Phi+\pi / 2$. We refer to this as normalized response or sensitivity.

If the sensitivity of the first harmonic does not depend on peak linear acceleration, it is possible to pool together trials with the same frequency but different peak accelerations. This was confirmed by a linear regression analysis (frequency and acceleration as regressors) performed on a subset of the data (all recordings with LED far and on, 16 recordings). The average coefficient for frequency was 0.7 and a $t$ test gave a $p$ value of $3 \times 10^{-7}$. The average coefficient for acceleration was 0.004 , resulting in a $p$ value of 0.81 . Telford et al. (1997), looking at linear VOR (IVOR) responses in monkeys, also concluded that to describe the main features of the lVOR responses, it was possible to average over acceleration amplitudes. Baarsma and Collewijn (1975) found the same in rabbits.

Averaged scores. Since the normalized response only changes with stimulus frequency, we calculated average response parameters of all conditions having the same frequency for each subject. To take the average, we went back to the vector representation of the response and calculated the average vector. From the average vector, we again extracted the corresponding magnitude and phase.

Relation between conjugate vertical ocular movements and cyclovergence. The components that modulated most were disconjugate torsional (cyclovergence) and conjugate vertical. To characterize the relation between the two, we computed the principal component in principal component analysis. The principal component corresponds to the direction with the maximum variance in two-dimensional scatter plots of conjugate vertical versus cyclovergence. This direction is given in degrees, with zero representing maximum variance along the vertical component. Therefore, $0^{\circ}$ is interpreted as absence of cyclovergence related to the vertical component; reciprocally, $90^{\circ}$ would indicate cyclovergence in the absence of a vertical component.

Quick phases of nystagmus. The direction of maximum variance in the scatter plots of cyclovergence and vertical was also computed for quick phases occurring during vestibular stimulation. Quick phases were isolated manually and represented by the calculated angular velocity rotation axis. For each quick phase, we determined the cyclovergence and vertical component and subsequently the direction of maximum variance for the scatter plot.

Estimated torsional modulation. Previous studies of cyclovergence reported the amplitude of torsional position. Since we analyzed angular velocity, we obtained an approximated value of torsional modulation $\theta_{\mathrm{DIS}} \approx\left(R_{\mathrm{DIS}} / 2 \pi f\right)$. We also estimated the amplitude of the conjugate

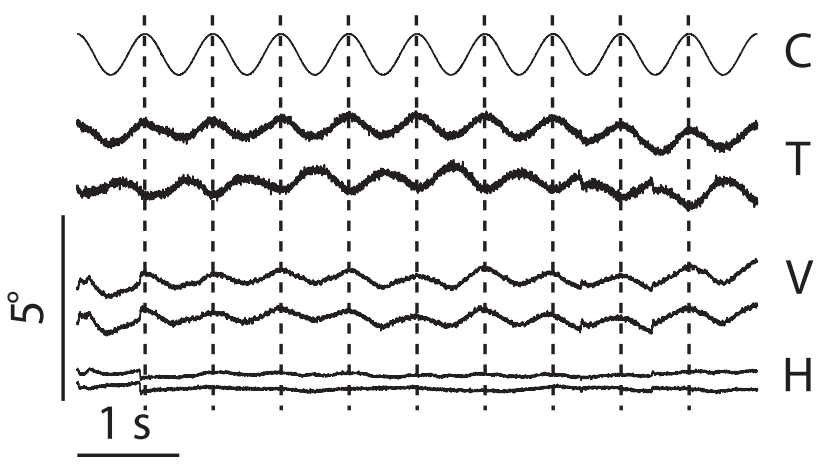

Figure 1. Typical traces of binocular rotation vectors. The magnitude of the rotation vector has been transformed to degrees of rotated angle. Traces have been displaced vertically to help visualization. The top trace of each component $(\mathrm{T}$, torsion; $\mathrm{V}$, vertical; $\mathrm{H}$, horizontal) corresponds to the right eye and the bottom to the left eye. The topmost trace $(C)$ shows the chair's vertical position (positive values up). Effective $\mathrm{g}$-load is maximal when the chair is at the bottom ( $\mathrm{g}$ load $=g+a$ ) and minimum at the top ( $g$-load $=g-a$ ), as explained in the Materials and Methods. As the chair goes up, decreasing g-load, both eyes move down (increased vertical component), and they extort. Stimulus was at $1.75 \mathrm{~Hz}$ and $0.7 \times g$ peak inertial linear acceleration. The LED was $143 \mathrm{~cm}$ straight ahead and continuously on. Dashed vertical lines indicate times when the chair was at its highest position.

vertical amplitude as $\theta_{\mathrm{VER}} \approx\left(R_{\mathrm{VER}} / 2 \pi f\right)$. To give an idea of the amplitudes of eye movements, we calculated the grand median over all subjects and trials corresponding to paradigm 1 in upright position with the fixation point far and constantly on.

\section{Results}

The size of cyclovergence was small; the median of estimated amplitude over all trials in upright position with frequencies between 1.5 and $2 \mathrm{~Hz}$ was $0.5^{\circ}$ and the amplitude of conjugate vertical $0.2^{\circ}$. Rotation vectors for both eyes as well as chair position traces are shown in Figure 1 for a typical trial in upright position with the far LED constantly on. Eyes extorted for chair up (downward acceleration, less effective gravity) and intorted for down (upward acceleration, stronger effective gravity) positions, and the torsional component was almost in phase with chair position.

For further analysis, we did not consider the components of rotation vectors, which describe position, but the components of angular velocity, which are independent of the chosen reference position. Figure 2 shows median angular velocity for a typical trial. Instead of right eye and left eye components, conjugate and disconjugate components are shown. As was apparent in Figure 1 , the main modulating components are the disconjugate component for torsion, i.e., cyclovergence, and the conjugate component for vertical.

In the following, we refer to normalized responses or sensitivities, in which the amplitude and phase of the angular velocity modulation are referenced to the amplitude and phase of inertial acceleration of the head as explained in Materials and Methods.

Figure 3 shows the interindividual averages and 95\% confidence intervals for the three experimental conditions. The broad spread in phase values observed at $1 \mathrm{~Hz}$ is due to the low amplitude of the angular velocity signal (and resulting decrease in signal-to-noise ratio). There was a significant increase of normalized amplitudes of cyclovergence and vertical eye movements with increasing frequency. The change in normalized amplitude from 1.5 to $2 \mathrm{~Hz}$ (mean $\pm 95 \% \mathrm{CI}$ ) in upright position with a far target was $4 \pm 1 \% \mathrm{~s} / \mathrm{g}$ for cyclovergence and $0.8 \pm 0.5 \% \mathrm{~s} / \mathrm{g}$ for vertical. With the near target, the differences were $3 \pm 1 \% \mathrm{~s} / \mathrm{g}$ for cyclovergence and $3.4 \pm 1.6^{\circ} / \mathrm{s} / g$ for vertical. Finally, in right ear 
down position, we found $4 \pm 2 \% \mathrm{~s} / \mathrm{g}$ for cyclovergence and $1.0 \pm 0.6 \% \mathrm{~s} / \mathrm{g}$ for vertical.

The change of phase with frequency depended on the paradigm. In upright position with far targets, the phase of cyclovergence increased significantly with frequency. The difference between phase at $1.5 \mathrm{~Hz}$ and phase at $2 \mathrm{~Hz}$ was $4 \pm 2^{\circ}$. The change in the phase of the vertical component was not statistically significant $-5 \pm$ $11^{\circ}$. In upright position with the near LED, the observed differences were not significant; the difference was $3 \pm 5^{\circ}$ for cyclovergence and $-4 \pm 8^{\circ}$ for vertical. The same was true for subjects in right ear down side position, in which we found $11 \pm$ $20^{\circ}$ for cyclovergence and $1 \pm 7^{\circ}$ for vertical.

The increase in the sensitivity of vertical eye movements with frequency probably reflects the corresponding decreased effectiveness of the pursuit system in the cancellation of the lVOR. On average, the vertical component of angular velocity was slightly phase advanced with respect to chair linear velocity. When pooling trials during UP-far with frequencies $>1 \mathrm{~Hz}$, the phase (mean $\pm 95 \% \mathrm{CI}$ ) for conjugate vertical was $-9 \pm 7^{\circ}$ and that of cyclovergence $8 \pm 2^{\circ}$.

When the LED was moved from $143 \mathrm{~cm}$ straight ahead to $\sim 16$ $\mathrm{cm}$ in front of the left eye, the conjugate vertical component showed an increase with the change in vergence; the mean increase in trials sharing the same inertial stimulus parameters was $11 \pm 3 \%$ s. Cyclovergence, however, remained at a level similar to that for far viewing.

With subjects lying on the side ( $90^{\circ}$ right ear down), we also observed disjunctive modulation of both eyes, although in some subjects there was also a small conjugate component. The small conjugate component, however, could have come from small roll head movements within the fields. Because of the swing of the GIV in this position, head motion could not be suppressed as completely as in upright position. As shown in Figure 3, cyclovergence in the right ear down position (data represented with circles) was comparable to that observed in the body upright position. Mean cyclovergence amplitudes and 95\% CI at the frequencies tested were $9 \pm 4,10 \pm 4$, and $13 \pm 6 \% \mathrm{~s} / \mathrm{g}$ at $1.5,1.75$, and $2 \mathrm{~Hz}$, respectively.

We next assessed whether cyclovergence modulation was related to Listing's law. For far targets, if there is compliance with Listing's law, cyclovergence and vertical angular velocity components are related by $\omega_{\mathrm{DIS}} \approx 2 \omega_{\mathrm{VER}} \sin \left(\theta_{\mathrm{DP}}\right)$. With targets at $1.4 \mathrm{~m}$, $\theta_{\mathrm{DP}}$, the temporal deviation of DP with respect to the nasooccipital axis, is small, $\theta_{\mathrm{DP}}<2.5^{\circ}$, and thus we would expect $\omega_{\mathrm{DIS}}$ $<0.1 \omega_{\text {VER }}$. We took two approaches. One consisted of comparing trials in which the target was constantly switched on with trials in which it was flashing. We did this to find out whether, during cancellation of the translational VOR response, the vertical component is better suppressed than the cyclovergence component. If this were the case, the relative size of both components should change and reach values consistent with Listing's law during trials with the flashing target. Both cyclovergence and vertical sensitivities increased slightly, by $2 \pm 1 \% \mathrm{~s} / \mathrm{g}$ from 10 to $12 \% \mathrm{~s} / \mathrm{g}$ and by $2 \pm 1 \% \mathrm{~s} / g$ from 4 to $6 \% \mathrm{~s} / g$, respectively. Even with a flashing target, the cyclovergence component was still about twice the size of the vertical, and thus much larger than that expected from Listing's law for a target at that distance $\left(\omega_{\text {DIS }}<\right.$ $\left.0.1 \omega_{\mathrm{VER}}\right)$. In the second approach, we compared the relation be- tween the cyclovergence and vertical components obtained from the slow-phase angular velocity to those obtained from the saccadic (quick) phases during vestibular stimulation. If cyclovergence is a result of Listing's law, we expect that the directions defined by the principal component of the scatter plots in both situations are close. The principal directions for slow phases and quick phases were calculated for each subject. The average and 95\% CI of the orientation of the principal direction for quick phases was $14.1 \pm 3.0^{\circ}$ and for slow phases $71.1 \pm 6.7^{\circ}$. Therefore, the principal direction was close to the vertical eye movement axis for quick phases and closer to the cyclovergence axis for slow phases. As long as eye orientation stays in DP, quick phases also stay in DP except for small transient blips (Straumann et al., 1995). Therefore, if slow-phase cyclovergence was the result of Listing's law, it should show a comparable orientation. Since this is not the case, this is further evidence that cyclovergence cannot be explained by Listing's law.

Last, we assessed whether smooth pursuit contributed to cyclovergence. In all the experimental conditions presented above, subjects were presented with an LED in a position fixed with respect to the head. In such cancellation paradigms, the LED light provides a pursuit signal that counters the vertical eye movement that would be evoked by the linear VOR in the dark. Figure 4 shows that cyclovergence was mainly related to the vestibular stimulus. Despite differences in the amplitude of the vertical component of angular eye velocity (circles, top row), cyclovergence amplitude (squares, bottom row) was comparable in all conditions with vestibular stimulation (all but C2). While cyclovergence amplitude during pursuit ranged from 0.1 to $0.6 \%$, during vestibular stimulation it ranged from 2.2 to $7.0 \%$ s. We thus conclude that the main contribution to cyclovergence is of vestibular origin.

\section{Discussion}

During sinusoidal up-down inertial acceleration with a fixation target that minimizes changes in gaze direction, we found cyclovergence. The normalized response (amplitude divided by inertial acceleration) of cyclovergence showed an increase with frequency, and the phase with respect to inertial acceleration averaged $8^{\circ}$.

\section{Characteristics of cyclovergence}

With upright subjects, we found dynamic excyclovergence when the effective gravity load decreased. This is opposite the results of de Graaf et al. (1996), but in the same direction as those reported by Clarke et al. (2007) for static cyclovergence in weightlessness. Merfeld et al. (1996) used sinusoidal earth-horizontal inertial acceleration, which generates a swinging GIV in the sagittal plane when subjects lay supine. Since in our experiments the GIV 


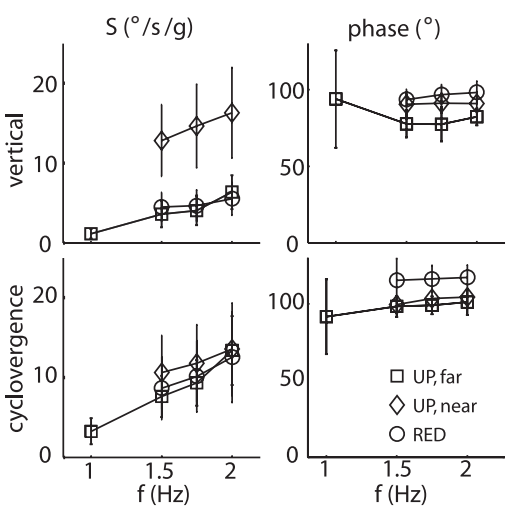

Figure 3. Interindividual averages and $95 \%$ confidence intervals for response sensitivity (left column) and phase (right column). Sensitivity is measured as the peak angular velocity divided by peak inertial acceleration and phase is between angular velocity and inertial acceleration (positive values signal a lag with respect to chair acceleration). For vertical eye movements, the phase for perfect compensation would be $90^{\circ}$. Averages are given for different experimental conditions. Squares, Subjects in upright position and LED $143 \mathrm{~cm}$ straight ahead; diamonds, upright position and LED $16 \mathrm{~cm}$ in front of the left eye; circles, subjects $90^{\circ}$ right ear down and LED $80 \mathrm{~cm}$ straight ahead.

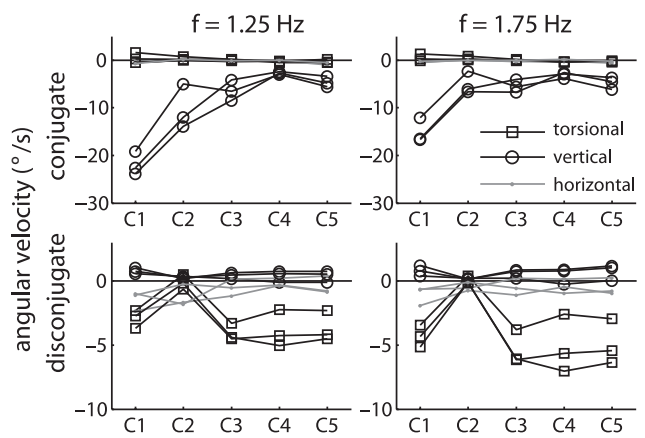

Figure 4. Dynamic cyclovergence is related to linear vestibular stimulation. The figure represents data from three subjects. C1 to C5 refer to the five visuovestibular conditions tested. With a target at a distance of $120 \mathrm{~cm}$, there were four conditions: C1, visually enhanced IVOR; $C 2$, pursuit with a stationary subject; C3, IVOR in complete darkness; $(4$, IVOR cancellation. The fifth condition, C5, was an IVOR cancellation paradigm with the target $60 \mathrm{~cm}$ away from the subject. The amplitude of the sinusoidal fit is shown in the top row for conjugate components and in the bottom row for the disconjugate components. Responses are considered negative when they are in counterphase to head motion in vestibular conditions, and in phase with target motion in smooth pursuit conditions. Stimuli were presented at two frequencies: $1.25 \mathrm{~Hz}$, shown in the left column, and $1.75 \mathrm{~Hz}$, in the right column.

changed only in magnitude, not direction, we conclude that the swing of the GIV in the sagittal plane cannot be the only source of cyclovergence. When we compare our data with that of Merfeld et al. (1996) (their Table 1), it seems that we find a smaller amplitude. Due to considerably different methodology, however, we do not know if this difference is significant.

Merfeld et al. (1996) also tested linear acceleration along the body vertical with subjects in the right ear down position and found cycloversion, which they assigned to the swinging of the GIV in the coronal plane. In the higher frequency range explored here, we found that cyclovergence in the right ear down position was similar to that in upright position (Fig. 3). It is possible that with the lower-frequency stimuli used by Merfeld et al. (1996), cyclovergence was either not produced or so small relative to cycloversion that it could not be appreciated. It could be that low-frequency cyclovergence is associated with the swing of the GIV in the sagittal plane. However, pitch reorientations fail to evoke cyclovergence (Diamond et al., 2006). The discrepancies

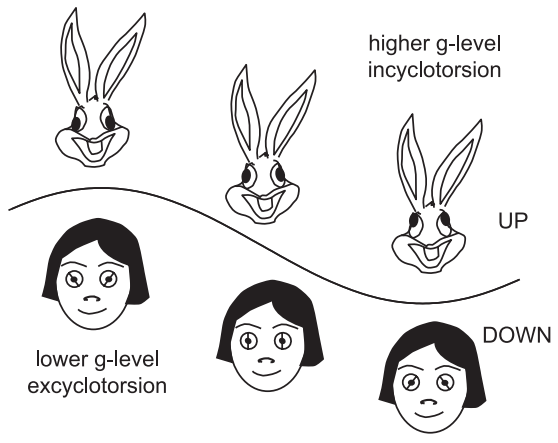

Figure 5. During up-down inertial acceleration, lateral-eyed species show a small modulation of the angle of elevation of the eye with respect to the horizontal horizon. In the head frame of reference, this is equivalent to a disjunctive torsional modulation of a frontal-eyed species. Eyes going down/up for the rabbit would correspond to eyes extorting/intorting for the frontal-eyed species. The figure represents the modulation during half a cycle of up-down sinusoidal motion and the expected modulation at three points: the highest point (lowest $g$-load), the intermediate point (normal g-load), and the lowest point (highest g-load).

among these studies could be due to the different frequency ranges tested and the effects of different orientation of the head relative to the pull of gravity.

Fixation targets moving with the subject are used to minimize horizontal and vertical eye movements and isolate torsion. Since the suppression of horizontal and vertical eye movements is achieved by the smooth pursuit system, it was possible that cyclovergence was not vestibular but associated with the pursuit command. Our control recordings in three stationary subjects showed no cyclovergence associated with pursuit of far targets (Fig. 4), which points to a purely vestibular origin of the cyclovergence we found in response to head translation.

We also found that cyclovergence during head translation had a similar size when fixating far and near targets $(143,80$, or 16 $\mathrm{cm})$. This is in contrast with the characteristics of the linear VOR response in foveate species, in which the amplitude of the eye movement is modulated by the object's distance (Schwarz et al., 1989; Paige et al., 1998).

\section{What is the origin of cyclovergence?}

Extended Listing's law

Cyclovergence evoked by motion along the vertical body axis with almost complete gaze stabilization and viewing of far targets is not consonant with our current understanding of what should be the eye movement response to head translation. Cyclovergence is only expected when focusing on near objects and accompanying larger vertical or vergence eye movements as determined by the temporal rotation of DPs of the right and left eyes upon convergence (Mok et al., 1992; Van Rijn and Van den Berg, 1993).

Clarke and Haslwanter (2007) found that, when the g-load changed from $1 \times g$ to $0 \times g$, the displacement plane of both eyes pitched backward and rotated temporally. These changes were more robust and bigger than those seen in humans during static pitch reorientations (Bockisch and Haslwanter, 2001). Since our stimulus was a dynamic change in gravity load (e.g., $0.5 \times g$ to $1.5 \times g$ when up-down inertial acceleration is $0.5 \times g)$, it is possible that the orientation of DP modulates during up-down motion. DP modulates during earth-horizontal yaw VOR in monkeys, where the torsional component of fast phases depends on the position of the head with respect to gravity (Hess and Angelaki, 1997a,b). Although we cannot exclude that the orientation of DP is modulating in response to the change in effective gravity load, the predicted relative size of the cyclovergence and 
vertical components of the slow phase would still be too small $\left(\omega_{\mathrm{DIS}}<0.1 \omega_{\mathrm{VER}}\right)$ to account for the cyclovergence found here.

\section{Relation to cycloversion}

Conjugate torsional eye movements in humans are elicited during inertial interaural stimulation and have low-pass frequency characteristics (Lichtenberg et al., 1982). Since the utricular maculae are not perfectly horizontal (Naganuma et al., 2003), the same mechanism that generates conjugate torsion during interaural acceleration could in principle generate cyclovergence during body-vertical acceleration and so contribute a low-pass frequency component to the cyclovergence found here. However, in the range of frequencies explored here, we did not find a reduction of amplitude with frequency. Therefore, we propose that another source of cyclovergence exists, which we term dynamic cyclovergence, originates from stimulation of the saccules and, like the translational VOR, is evoked by high-frequency translations along the vertical axis of the head (Paige, 1989; Liao et al., 2008a,b).

\section{Phylogenetic origin}

An intriguing possibility is that the cyclovergence described here is a relic from the VOR response of lateral-eyed animals to vertical translation, just as the small conjugate torsional eye movements observed in frontal-eyed animals during interaural translation have been interpreted as a phylogenetic remnant of the response of lateral-eyed animals to linear acceleration (Brodsky, 2002).

Otolith-ocular reflexes in lateral-eyed species are comprised mainly of tilt responses (Baarsma and Collewijn, 1975; Maruta et al., 2001), but there are also small horizontal eye movements in response to interaural translation and vertical responses to highfrequency vertical stimuli (Hess and Dieringer, 1991). Although the latter have only been looked for in rats, it is possible that a rudimentary vertical linear VOR response is also present in other lateral-eyed animals. Compensatory ocular responses to vertical translation, though best defined in an eye-referenced frame, are likely implemented using the natural head-referenced frame provided by the semicircular canals, as has been suggested for smooth pursuit (FitzGibbon et al., 1996). If the same basic central circuitry survives in frontal-eyed animals, the resulting eye motion would correspond to cyclovergence (Fig. 5) as observed here, with elevation accompanied by intorsion and vice versa. This is because eye movement commands encoded in this reference frame lead to the same head-centered eye movements in all species even when the orientation of the semicircular canals, the optic axis, and extraocular muscle insertions into the globe vary across species. In this way, the same basic circuitry evokes eye movements compensatory for head rotations in lateral- and frontal-eyed species (Simpson and Graf, 1981).

The same idea applies to the apparent tilt responses observed during linear translations in the horizontal plane, which lead to a swing of the gravitoinertial axis equivalent to that of a head tilt. Apparent tilt responses are functional and of considerable size in lateral-eyed species (Baarsma and Collewijn, 1975; Maruta et al., 2001), but in frontal-eyed species they would interfere with stereopsis and are necessarily small (Collewijn et al., 1985; Paige and Tomko, 1991a,b; Angelaki, 1998; Misslisch et al., 2001; Brodsky, 2002). Disease may uncover these suppressed circuits, as exemplified by the ocular tilt reaction, which includes skew deviation, binocular cyclorotation, and roll tilt of the head, "a phylogenetically old righting response to a lateral tilt of the head" (e.g., Zee, 1996).

Animals who are partially lateral-eyed also display appropriate compensatory responses to translational motion. In the case of naso-occipital and interaural translations and a straight-ahead target, the requirements are the same for frontal and predominantly lateral-eyed species: vergence for naso-occipital and horizontal ocular responses for interaural translations. Both types are present in frogs (Straka and Dieringer, 2004), rats (Hess and Dieringer, 1991), and monkeys (e.g., Paige and Tomko, 1991).

For a straight-ahead target, only the components of translation along the vertical axis of the head require different compensatory eye movements in lateral- and frontal-eyed species. We speculate that the phylogenetically old central circuitry survives to some extent in parallel to an evolutionarily newer circuitry subserving the translational VOR of foveate species, where proper horizontal and torsion alignment become important for stereopsis. The cerebellum likely plays a role in the implementation of the necessary adjustments in the compensatory response to translation based on the needs of the fovea and successful binocular vision (Zee et al., 2002).

\section{Summary}

We found cyclovergence during relatively high-frequency sinusoidal earth-vertical linear motion. This cyclovergence contains a high-pass contribution that, unlike ocular counterroll or the lowpass component of cyclovergence, is likely related to the translational VOR and not the orienting VOR. We propose that the high-pass contribution depends on the saccules and originates in the phylogenetically older response of lateral-eyed animals to vertical inertial stimulation.

\section{References}

Angelaki DE (1998) Three-dimensional organization of otolith-ocular reflexes in rhesus monkeys. III. Responses to translation. J Neurophysiol 80:680-695.

Baarsma EA, Collewijn H (1975) Eye movements due to linear accelerations in the rabbit. J Physiol 245:227-247.

Bockisch CJ, Haslwanter T (2001) Three-dimensional eye position during static roll and pitch in humans. Vision Res 41:2127-2137.

Brodsky MC (2002) Do you really need your oblique muscles? Adaptations and exaptations. Arch Ophthalmol 120:820-828.

Cheung BS, Howard IP (1991) Optokinetic torsion: dynamics and relation to circularvection. Vision Res 31:1327-1335.

Clarke AH, Haslwanter T (2007) The orientation of Listing's plane in microgravity. Vision Res 47:3132-3140.

Collewijn H, Van der Steen J, Ferman L, Jansen TC (1985) Human ocular counterroll: assessment of static and dynamic properties from electromagnetic scleral coil recordings. Exp Brain Res 59:185-196.

Curthoys IS, Burgess AM, MacDougall HG, McGarvie LA, Halmagyi GM, Smulders YE, Iwasaki S (2009) Testing human otolith function using bone-conducted vibration. Ann N Y Acad Sci 1164:344-346.

de Graaf B, Bos JE, Groen E (1996) Saccular impact on ocular torsion. Brain Res Bull 40:321-326; discussion 326-30.

Diamond SG, Markham CH (1998) Changes in gravitational state cause changes in ocular torsion. J Gravit Physiol 5:P109-P110.

Diamond SG, Markham CH, Clarke AH (2006) Dynamic pitch rotation affects eye torsion. Acta Otolaryngol 126:248-253.

FitzGibbon EJ, Calvert PC, Dieterich M, Brandt T, Zee DS (1996) Torsional nystagmus during vertical pursuit. J Neuroophthalmol 16:79-90.

Haustein W (1989) Considerations on Listing's law and the primary position by means of a matrix description of eye position control. Biol Cybern 60:411-420.

Helmholtz H (1867) Handbuch der physiologischen Optik. Leipzig: Voss.

Hess BJ, Angelaki DE (1997a) Kinematic principles of primate rotational vestibulo-ocular reflex. I. Spatial organization of fast phase velocity axes. J Neurophysiol 78:2193-2202.

Hess BJ, Angelaki DE (1997b) Kinematic principles of primate rotational vestibulo-ocular reflex II. Gravity-dependent modulation of primary eye position. J Neurophysiol 78:2203-2216.

Hess BJ, Dieringer N (1991) Spatial organization of linear vestibuloocular reflexes of the rat: responses during horizontal and vertical linear acceleration. J Neurophysiol 66:1805-1818. 
Liao K, Walker MF, Joshi A, Reschke M, Leigh RJ (2008a) Vestibulo-ocular responses to vertical translation in normal human subjects. Exp Brain Res 185:553-562.

Liao K, Walker MF, Joshi A, Reschke M, Wang Z, Leigh RJ (2008b) A reinterpretation of the purpose of the translational vestibulo-ocular reflex in human subjects. Prog Brain Res 171:295-302.

Lichtenberg BK, Young LR, Arrott AP (1982) Human ocular counterrolling induced by varying linear accelerations. Exp Brain Res 48:127-136.

Markham CH, Diamond SG (2002) Ocular counterrolling in response to static and dynamic tilting: implications for human otolith function. J Vestib Res 12:127-134.

Maruta J, Simpson JI, Raphan T, Cohen B (2001) Orienting otolith-ocular reflexes in the rabbit during static and dynamic tilts and off-vertical axis rotation. Vision Res 41:3255-3270.

Merfeld DM, Teiwes W, Clarke AH, Scherer H, Young LR (1996) The dynamic contributions of the otolith organs to human ocular torsion. Exp Brain Res 110:315-321.

Merfeld DM, Park S, Gianna-Poulin C, Black FO, Wood S (2005) Vestibular perception and action employ qualitatively different mechanisms. II. VOR and perceptual responses during combined Tilt\&Translation. J Neurophysiol 94:199-205.

Misslisch H, Tweed D, Hess BJ (2001) Stereopsis outweighs gravity in the control of the eyes. J Neurosci 21:RC126.

Mok D, Ro A, Cadera W, Crawford JD, Vilis T (1992) Rotation of Listing's plane during vergence. Vision Res 32:2055-2064.

Moore ST, Hirasaki E, Raphan T, Cohen B (2001) The human vestibuloocular reflex during linear locomotion. Ann N Y Acad Sci 942:139-147.

Naganuma H, Tokumasu K, Okamoto M, Hashimoto S, Yamashina S (2003) Three-dimensional analysis of morphological aspects of the human utricular macula. Ann Otol Rhinol Laryngol 112:419-424.

Olasagasti I, Bockisch CJ, Zee DS, Straumann D (2008) Cyclovergence evoked by up-down acceleration along longitudinal axis in humans. Prog Brain Res 171:319-322.

Paige GD (1989) The influence of target distance on eye movement responses during vertical linear motion. Exp Brain Res 77:585-593.

Paige GD, Tomko DL (1991a) Eye movement responses to linear head motion in the squirrel monkey. I. Basic characteristics. J Neurophysiol 65:1170-1182.
Paige GD, Tomko DL (1991b) Eye movement responses to linear head motion in the squirrel monkey. II. Visual-vestibular interactions and kinematic considerations. J Neurophysiol 65:1183-1196.

Paige GD, Telford L, Seidman SH, Barnes GR (1998) Human vestibuloocular reflex and its interactions with vision and fixation distance during linear and angular head movement. J Neurophysiol 80:2391-2404.

Palla A, Bockisch CJ, Bergamin O, Straumann D (2006) Dissociated hysteresis of static ocular counterroll in humans. J Neurophysiol 95:2222-2232.

Pansell T, Ygge J, Schworm HD (2003) Conjugacy of torsional eye movements in response to a head tilt paradigm. Invest Ophthalmol Vis Sci 44:2557-2564.

Raphan T, Cohen B (2002) The vestibulo-ocular reflex in three dimensions. Exp Brain Res 145:1-27.

Savitzky A, Golay MJE (1964) Smoothing and differentiation of data by simplified least squares procedures. Anal Chem 36:1627-1639.

Schwarz U, Busettini C, Miles FA (1989) Ocular responses to linear motion are inversely proportional to viewing distance. Science 245:1394-1396.

Simpson JI, Graf W (1981) Eye-muscle geometry and compensatory eye movements in lateral-eyed and frontal-eyed animals. Ann N Y Acad Sci 374:20-30

Straka H, Dieringer N (2004) Basic organization principles of the VOR: lessons from frogs. Prog Neurobiol 73:259-309.

Straumann D, Zee DS, Solomon D, Lasker AG, Roberts DC (1995) Transient torsion during and after saccades. Vision Res 35:3321-3334.

Telford L, Seidman SH, Paige GD (1997) Dynamics of squirrel monkey linear vestibuloocular reflex and interactions with fixation distance. J Neurophysiol 78:1775-1790.

Van Rijn LJ, Van den Berg AV (1993) Binocular eye orientation during fixations: Listing's law extended to include eye vergence. Vision Res 33:691-708

Zee DS (1996) Considerations on the mechanisms of alternating skew deviation in patients with cerebellar lesions. J Vestib Res 6:395-401.

Zee DS, Walker MF, Ramat S (2002) The cerebellar contribution to eye movements based upon lesions: binocular three-axis control and the translational vestibulo-ocular reflex. Ann N Y Acad Sci 956:178-189.

Zupan LH, Merfeld DM (2005) Human ocular torsion and perceived roll responses to linear acceleration. J Vestib Res 15:173-183. 